\title{
Visit-to-visit variability in blood pressure over a 1 -year period is a marker of left ventricular diastolic dysfunction in treated hypertensive patients
}

\author{
Hisashi Masugata $^{1}$, Shoichi Senda ${ }^{1}$, Koji Murao ${ }^{2}$, Michio Inukai ${ }^{1}$, Naohisa Hosomi ${ }^{3}$, Yasuyoshi Iwado ${ }^{4}$, \\ Takahisa Noma ${ }^{4}$, Masakazu Kohno ${ }^{4}$, Takashi Himoto ${ }^{1}$ and Fuminori Goda ${ }^{1}$
}

Although visit-to-visit variability in systolic blood pressure (SBP) has recently been demonstrated to be a strong predictor of stroke, there are no data about relationships between SBP variability and cardiac damage in hypertensive patients. We compared relationships between visit-to-visit variability in SBP and left ventricular (LV) diastolic dysfunction with the relationships between the mean SBP value and cardiac parameters in treated patients. Forty treated hypertensive patients (69 \pm 9 years of age) had their blood pressure measured at outpatient clinics every 1 or 2 months over a 1 -year period. The standard deviation (s.d.) of SBP and the difference between the maximum and minimum SBPs during this year were calculated to assess visit-to-visit variability. The mean SBP during the year was also calculated. LV diastolic function was assessed by the ratio (E/A) of early (E) and late (A) diastolic transmitral flows, early diastolic mitral annular velocity ( $\left.e^{\prime}\right)$ and the ratio (E/e') of $E$ to $e^{\prime}$ using Doppler echocardiography. E/A only correlated with the s.d. of SBP $(r=-0.327, P=0.040)$, whereas $\mathrm{e}^{\prime}$ correlated with s.d. of SBP $(r=-0.496, P=0.001)$ and maximum-minimum SBP difference $(r=-0.490, P=0.001)$. E/e' correlated with s.d. of SBP ( $r=0.384, P=0.014)$, maximum-minimum SBP difference $(r=0.410, P=0.009)$, and the mean value of SBP $(r=0.349$, $P=0.028$ ). Multiple regression analysis demonstrated only the maximum-minimum SBP difference independently associated with $E / e^{\prime}(\beta=0.410, P=0.009)$. Thus, the visit-to-visit variability of SBP showed better correlation with LV diastolic dysfunction than mean values of SBP. High visit-to-visit variability of SBP was associated with LV diastolic dysfunction and may constitute a high risk for diastolic heart failure in hypertensive patients.

Hypertension Research (2011) 34, 846-850; doi:10.1038/hr.2011.54; published online 12 May 2011

Keywords: left ventricular diastolic dysfunction; seasonal variation; variability of blood pressure

\section{INTRODUCTION}

Left ventricular (LV) hypertrophy and diastolic dysfunction are commonly observed in hypertensive patients ${ }^{1,2}$ and are risk factors of chronic heart failure due to LV diastolic dysfunction..$^{3-6}$ Patients with chronic heart failure due to LV diastolic dysfunction have apparent preservation of systolic function, defined by an ejection fraction $\geqslant 50 \%{ }^{7,8}$ Such patients are typically obese females, and they often have a history of chronic hypertension and LV hypertrophy. One of the important characteristics of heart failure due to LV diastolic dysfunction is that it is often observed in elderly hypertensive patients. ${ }^{9}$ This is likely because arterial stiffening due to aging or hypertension leads to LV hypertrophy or LV interstitial fibrosis, thereby producing $\mathrm{LV}$ diastolic dysfunction. ${ }^{10,11}$

Seasonal variation in arterial blood pressures has been demonstrated; ${ }^{12-19}$ blood pressures are higher in the winter than in the summer. Studies in older adults ${ }^{20,21}$ have shown that a $10-\mathrm{mm} \mathrm{Hg}$ rise in systolic blood pressure (SBP) is associated with an approximately $10 \%$ increase in the risk of death from stroke and ischemic heart disease. Visit-to-visit variability in SBP (standard deviation (s.d.) of SBP) has recently been demonstrated to be a strong predictor of stroke, independent of the mean value of SBP. ${ }^{22,23}$ However, there are no data about relationships between SBP variability and cardiac damage in hypertensive patients.

We hypothesized that visit-to-visit SBP variability over a 1-year period may reflect cardiac damage, such as LV hypertrophy and diastolic dysfunction, better than the mean value of SBP during the same period. We also hypothesized that visit-to-visit SBP variability has a stronger correlation with LV hypertrophy and diastolic dysfunction, thereby more reliably reflecting cardiac damage than does mean SBP. In the present study, we examined the associations between two

${ }^{1}$ Department of Integrated Medicine, Kagawa University, Kagawa, Japan; ${ }^{2}$ Division of Endocrinology and Metabolism, Department of Internal Medicine, Kagawa University, Kagawa, Japan; ${ }^{3}$ Department of Clinical Neuroscience and Therapeutics, Hiroshima University Graduate School of Biomedical Sciences, Hiroshima, Japan and ${ }^{4}$ Department of Cardiorenal and Cerebrovascular Medicine, Kagawa University, Kagawa, Japan

Correspondence: Dr H Masugata, Department of Integrated Medicine, Kagawa University, 1750-1, Miki-cho, Kita-gun, Kagawa 761-0793, Japan.

E-mail: masugata@med.kagawa-u.ac.jp

Received 26 November 2010; revised 22 January 2011; accepted 7 February 2011; published online 12 May 2011 
parameters of visit-to-visit SBP variability over a 1-year period and LV hypertrophy and diastolic dysfunction in treated hypertensive patients. These associations were compared with those of mean SBP.

\section{METHODS}

\section{Subjects and protocol}

The study subjects were 40 patients (19 male, 21 female; mean age $69 \pm 9$ years, range 50-83 years) who had been diagnosed with hypertension at Kagawa University Hospital and who had regularly visited the outpatient clinic from September 2009 through September 2010. Hypertension was defined as SBP $\geqslant 140 \mathrm{~mm} \mathrm{Hg}$ and/or diastolic blood pressure (DBP) $\geqslant 90 \mathrm{~mm} \mathrm{Hg}$. Blood pressure was determined using the conventional cuff method. All patients were treated with at least one antihypertensive drug. For at least 1 year before their enrollment in this study, the antihypertensive drugs did not change for any patients. In addition, the antihypertensive drugs did not change for any patients over the 1-year observation period for obtaining blood pressure data. Patients with a history of heart failure or obvious heart disease were excluded. None of the patients had a history of atherosclerotic cardiovascular disease or stroke. Blood pressure was measured at an outpatient clinic every 1 or 2 months over the 1-year observation period for each patient. This study started the observation of blood pressure at September. At the end of the 1-year observation period, echocardiographic examinations were performed to assess cardiac structural changes and cardiac function. Thus, the echocardiography was performed at September in all patients. Arterial stiffness was assessed by measuring cardio-ankle vascular index (CAVI) just after the echocardiographic examination. In addition, blood samples were taken in the morning after an 8-h overnight fast. Plasma total cholesterol, triglyceride, high-density lipoprotein cholesterol, serum albumin, serum creatinine and hemoglobin were measured by standard laboratory techniques. Patients with renal dysfunction whose serum creatinine levels were $\geqslant 1.2 \mathrm{mg} \mathrm{dl}^{-1}$ were excluded from the present study. The relationships between echocardiographic parameters and various clinical characteristics, including blood pressure, CAVI and laboratory data, were analyzed. This protocol was approved by the Ethics Committee of Kagawa University, and informed consent was obtained from all participants.

\section{Echocardiographic examination}

Two-dimensional and M-mode echocardiographies were performed using the Vivid Seven System (GE Healthcare; Horten, Norway). We first measured the following LV structural parameters at the chordae tendineae level by M-mode echocardiography: ventricular septal thickness (VS), LV end-diastolic dimension (LVDd) and LV end-systolic dimension (LVDs) and LV posterior wall (PW) thickness. The LV mass was calculated according to the American Society of Echocardiography convention ${ }^{24}$ using the following formula: LV mass $=0.80 \times 1.04 \times\left((\mathrm{PW}+\mathrm{VS}+\mathrm{LVDd})^{3}-(\mathrm{LVDd})^{3}\right)+0.6$. The LV mass index (LVMI) was calculated as the LV mass divided by the body surface area. The LV ejection fraction was estimated by Teichholz's method ${ }^{25}$ and was used as the parameter of LV systolic function.

We next measured the parameters of LV diastolic function by recording the transmitral flow velocity using conventional Doppler echocardiography, which measures blood flow velocities in the cardiac cavity. ${ }^{26,27}$ The transmitral flow velocity was recorded from the apical transducer position with the sample volume situated between the mitral leaflet tips. The peak velocity of early transmitral flow velocity (E velocity) and the peak velocity of late transmitral flow velocity (A velocity) were recorded, and the E/A ratio was calculated as a parameter of LV diastolic function.

In addition, tissue Doppler echocardiography, which measures the velocities of the regional cardiac wall, was performed by activating the tissue Doppler echocardiographic function in the same machine. Mitral annular velocities were recorded from the apical window. Sample volumes were located at the septal site of the mitral annulus. Peak early diastolic mitral annular velocity $\left(\mathrm{e}^{\prime}\right)$ and the ratio of E velocity to $\mathrm{e}^{\prime}$ velocity $\left(\mathrm{E} / \mathrm{e}^{\prime}\right)$ were measured and analyzed as parameters of LV diastolic function. ${ }^{28,29}$

\section{Assessment of arterial stiffness by measuring cardio-ankle vascular index}

CAVI was measured using an automatic vascular screening system (VaseraVS1000; Fukuda Densi, Tokyo, Japan) with the patient resting in a supine position. Pulse wave velocity (PWV) was obtained by dividing the vascular length by the time it took for the pulse wave to propagate from the aortic valve to the ankle. SBP and DBP were measured at the brachial artery. The formula used to calculate CAVI is as follows: CAVI $=a((2 \rho / \Delta P) \times \ln \quad(\mathrm{SBP} / \mathrm{DBP})$ $\left.\mathrm{PWV}^{2}\right)+b$, where $\Delta P$ is SBP-DBP, $\rho$ is blood density and $a$ and $b$ are scaleconversion constants to match aortic PWV. The principle underlying CAVI has been described previously. ${ }^{30}$ The equation is derived from Bramwell-Hill's equation and the stiffness parameter $\beta$, and CAVI was adjusted for blood pressure, based on the stiffness parameter $\beta$. Therefore, CAVI reflects the stiffness of the aorta, femoral artery and tibial artery as a whole; theoretically, it is not affected by blood pressure. All these measurements and calculations were performed using a VaSera VS-1000 (Fukuda Densi). Electrocardiogram electrodes were placed on both wrists, a microphone for detecting heart sounds was placed on the sternum, and cuffs were wrapped around both arms and both ankles. After automatic measurements, the right and left CAVI values were calculated, and the averages of these values were used for analysis.

\section{Statistical analysis}

Data are expressed as means \pm s.d. Statistical analysis was performed using the SPSS software package (SPSS, Chicago, IL, USA). The parametric distribution of the variables was analyzed using the Kolmogorov-Smirnov test. Comparison between the groups was made using the Mann-Whitney test for data with nonnormal distribution. For the comparison of monthly mean values of SBP obtained from all subjects over the months, one-way repeated analysis of variance was used. Correlations among echocardiographic parameters, blood pressure and other variables were assessed by univariate analyses. Subsequently, using factors deemed significant in the univariate analyses, stepwise multiple regression analysis was performed to select factors independently associated with parameters of LV diastolic function. Values of $P<0.05$ were considered statistically significant

\section{RESULTS}

Clinical and echocardiographic characteristics of subjects

The clinical and echocardiographic parameters of the study subjects are summarized in Table 1 . The mean values of SBP $(129 \pm 12 \mathrm{~mm} \mathrm{Hg})$ and DBP $(70 \pm 9 \mathrm{~mm} \mathrm{Hg})$ over the 1-year observation period in all subjects was not particularly high because all patients' blood pressures were appropriately controlled by medication. The high mean value of CAVI $(9.0 \pm 1.5)$ indicated the presence of arterial stiffening in the subjects as a whole. The mean LV ejection fraction was $71 \pm 7 \%$; all patients had normal systolic function (LV ejection fraction $\geqslant 55 \%$ ). The elevated mean LVMI $\left(114 \pm 26 \mathrm{~g} \mathrm{~m}^{-2}\right)$ indicated the presence of LV hypertrophy in the subjects as a whole. In addition, the decreased mean E/A (0.78 \pm 0.18$)$, decreased $\mathrm{e}^{\prime}\left(5.6 \pm 1.4 \mathrm{~cm} \mathrm{~s}^{-1}\right)$ and increased $\mathrm{E} / \mathrm{e}^{\prime}(10.6 \pm 3.0)$ indicated LV diastolic dysfunction accompanying the LV hypertrophy.

\section{Visit-to-visit variability in SBP over the 1-year observation}

The visit-to-visit variability in SBP over the 1-year observation in all subjects is shown in Figure 1. As the SBP increased in the winter and decreased in the summer, this visit-to-visit variability in SBP indicated the seasonal variation of SBP. The highest level of SBP was observed in November, and the lowest level of SBP was observed in August. The monthly average SBP of the subjects in November was statistically higher than the value obtained in August $(P=0.044)$. The s.d. of SBP $(11 \pm 5 \mathrm{~mm} \mathrm{Hg})$ and maximum-minimum SBP $(31 \pm 16 \mathrm{~mm} \mathrm{Hg})$ over the 1-year observation in all subjects indicated the presence of considerably large visit-to-visit SBP variability (Table 1 ). The highest DBP level ( $75 \pm 11 \mathrm{~mm} \mathrm{Hg}$ ) was observed in November, and the lowest DBP $(67 \pm 10 \mathrm{~mm} \mathrm{Hg})$ was observed in July. The highest level of pulse pressure $(66 \pm 17 \mathrm{~mm} \mathrm{Hg}$ ) was observed in October, and the lowest level of pulse pressure $(55 \pm 13 \mathrm{~mm} \mathrm{Hg})$ was observed in September. However, the seasonal variations in DBP and pulse pressure were not statistically significant. 
Table 1 Clinical and echocardiographic characteristics of the subjects

\begin{tabular}{|c|c|}
\hline Number (male/female) & $40(19 / 21)$ \\
\hline Age (years) & $69 \pm 9$ \\
\hline $\mathrm{BMI}\left(\mathrm{kg} \mathrm{m}^{-2}\right)$ & $24.3 \pm 3.7$ \\
\hline Total cholesterol (mg dl-1) & $192 \pm 27$ \\
\hline HDL cholesterol (mg dl ${ }^{-1}$ ) & $53 \pm 13$ \\
\hline Triglyceride $\left(\mathrm{mg} \mathrm{dl}^{-1}\right)$ & $136 \pm 91$ \\
\hline Serum albumin $\left(\mathrm{g} \mathrm{dl}^{-1}\right)$ & $4.2 \pm 0.3$ \\
\hline Serum creatinin $\left(\mathrm{mg} \mathrm{dl}^{-1}\right)$ & $0.75 \pm 0.21$ \\
\hline $\mathrm{Hb}\left(\mathrm{gdl}^{-1}\right)$ & $13.1 \pm 1.4$ \\
\hline \multicolumn{2}{|l|}{ Drug administration } \\
\hline ARB (\%) & 73 \\
\hline CCB (\%) & 75 \\
\hline$\alpha$-Blocker (\%) & 3 \\
\hline$\beta$-Blocker (\%) & 20 \\
\hline Diuretic (\%) & 18 \\
\hline
\end{tabular}

Mean values of blood pressure and heart rate during 1-year observation

Mean value of SBP $(\mathrm{mm} \mathrm{Hg})$

$129 \pm 12$

Mean value of DBP $(\mathrm{mm} \mathrm{Hg})$

$70 \pm 9$

Mean value of $\mathrm{HR}$ (beats per min)

$68 \pm 9$

Visit-to-visit variability of SBP during 1-year observation

$$
\begin{aligned}
& \text { s.d. of SBP }(\mathrm{mm} \mathrm{Hg}) \\
& \text { Maximum-minimum SBP }(\mathrm{mm} \mathrm{Hg})
\end{aligned}
$$

$11 \pm 5$

$31 \pm 16$

CAVI

$9.0 \pm 1.5$

$$
\begin{aligned}
& \text { LV structure } \\
& \text { LVMI }\left(\mathrm{g} \mathrm{m}^{-2}\right) \\
& \text { LV systolic function } \\
& \text { LVEF (\%) } \\
& \text { LV diastolic function } \\
& \text { E/A } \\
& \mathrm{e}^{\prime}\left(\mathrm{cm} \mathrm{s}^{-1}\right) \\
& \text { E/ } \mathrm{e}^{\prime}
\end{aligned}
$$

$$
71 \pm 7
$$$$
114 \pm 26
$$

$0.78 \pm 0.18$

$5.6 \pm 1.4$

$10.6 \pm 3.0$

Abbreviations: e', peak early diastolic mitral annular velocity; A, peak late diastolic transmitral flow; ARB, angiotensin II receptor blocker; BMI, body mass index; CAVI, cardio-ankle vascular index; $\mathrm{CCB}$, calcium channel blocker; $\mathrm{DBP}$, diastolic brachial blood pressure; $\mathrm{Hb}$, hemoglobin; $H D L$, high-density lipoprotein; $H R$, heart rate; $E$, peak early diastolic transmitral flow; $E / A$, the ratio of $E$ to $A ; E / e^{\prime}$, the ratio of $E$ to $e^{\prime}$; LV, left ventricular; LVEF, left ventricular ejection fraction; LVMI, left ventricular mass index; SBP, systolic brachial blood pressure; s.d., standard deviation.

Association of blood pressure variation parameters and mean values of blood pressure with CAVI over the 1-year observation Linear regression analysis was performed to examine the relationship of blood pressure variation parameters and mean values of blood pressure with CAVI in all subjects over the 1-year observation (Table 2). The two parameters of visit-to-visit variability of SBP (s.d. of SBP and maximum-minimum SBP difference) closely correlated with each other $(r=0.958, P<0.001)$. Both the s.d. of SBP and the maximum-minimum SBP difference showed weak correlations with the mean value of SBP ( $r=0.353, P=0.025$ and $r=0.390, P=0.013$, respectively). CAVI did not show statistically significant correlations with mean values of blood pressure and visit-to-visit variability of SBP over the 1-year observation.

Comparison of visit-to-visit SBP variability parameters and mean SBP over the 1-year observation, in terms of their correlation with LV diastolic function

Linear regression analysis was performed to examine the relationships between parameters of visit-to-visit SBP variability, mean value of SBP

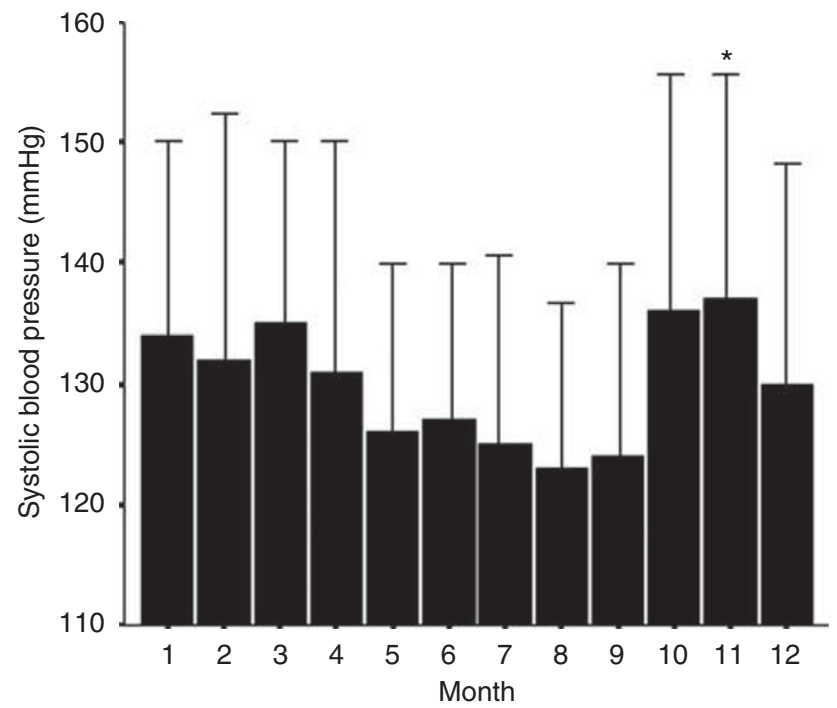

Figure 1 Visit-to-visit variability in systolic blood pressure (SBP) over a 1 -year observation, for all subjects. The SBP increased in the winter and decreased in the summer. The highest level of SBP was observed in November, and the lowest level was observed in August. The monthly mean value of SBP of the subjects in November was statistically higher than the value obtained in August $(P=0.044)$. ${ }^{*} P<0.05$ vs. August.

Table 2 Correlations between blood pressure variability, mean values of blood pressure during 1-year observation, and CAVI

\begin{tabular}{lccr}
\hline & SD of SBP & Max-min SBP difference & CAVI \\
\cline { 2 - 4 } Variable & $r$ & $r$ & $r$ \\
\hline Mean values of blood pressure and heart rate during & 1 -year observation & \\
Mean value of SBP & $0.353^{*}$ & $0.390^{*}$ & 0.227 \\
Mean value of DBP & 0.147 & 0.200 & -0.206 \\
Mean value of HR & 0.285 & 0.238 & -0.026 \\
& & & \\
Visit-to-visit variability of SBP & during 1-year observation & 0.304 \\
SD of SBP & - & $0.958^{* * *}$ & 0.303 \\
Max-min SBP difference & $0.958^{* * *}$ & - &
\end{tabular}

Abbreviations: DBP, diastolic brachial blood pressure; HR, heart rate; Max-min, maximumminimum; s.d., standard deviation; SBP, systolic brachial blood pressure. ${ }^{*} P<0.05, * * * P<0.001$

over the 1-year observation, and echocardiographic parameters in all subjects (Table 3). Among the blood pressure variables, only maximum-minimum SBP showed a significant correlation $(r=0.319$, $P=0.045)$ with LVMI, which is a parameter of LV hypertrophy, although the correlation was weak. Regarding parameters of LV diastolic function, both parameters of visit-to-visit SBP variability over the 1-year observation correlated with the parameters of LV diastolic function. However, parameters of mean values of blood pressure during the 1-year observation did not have a good correlation with parameters of LV diastolic function. The mean SBP correlated only with $\mathrm{E} / \mathrm{e}^{\prime}(r=0.349, P=0.028)$. In contrast, parameters of visit-tovisit SBP variability showed good correlations with the parameters of LV diastolic function. The s.d. of SBP was associated with E/A $(r=-0.327, P=0.040), \mathrm{e}^{\prime}(r=-0.496, P=0.001)$ and $\mathrm{E} / \mathrm{e}^{\prime} \quad(r=0.384$, $P=0.014)$. In addition, the maximum-minimum SBP difference was associated with $\mathrm{e}^{\prime}(r=-0.490, P=0.001)$ and $\mathrm{E} / \mathrm{e}^{\prime}(r=0.410, P=0.009)$. 
Table 3 Comparison of the correlations with parameters of left ventricular hypertrophy and diastolic function

\begin{tabular}{|c|c|c|c|c|}
\hline \multirow[b]{3}{*}{ Variable } & \multirow{3}{*}{$\begin{array}{l}\text { LV hypertrophy } \\
\text { LVMI } \\
r\end{array}$} & \multicolumn{3}{|c|}{ LV diastolic function } \\
\hline & & $E / A$ & $e^{\prime}$ & $E / e^{\prime}$ \\
\hline & & $r$ & $r$ & $r$ \\
\hline Age & 0.196 & $-0.468^{* *}$ & $-0.447^{* *}$ & 0.175 \\
\hline BMI & -0.044 & -0.012 & 0.196 & -0.037 \\
\hline Total cholesterol & 0.056 & 0.008 & 0.057 & -0.017 \\
\hline Triglyceride & 0.001 & -0.075 & -0.053 & 0.133 \\
\hline HDL cholesterol & -0.223 & 0.140 & 0.222 & -0.192 \\
\hline Serum creatinine & 0.253 & -0.311 & $-0.348^{*}$ & 0.007 \\
\hline Serum albumin & 0.200 & -0.093 & 0.180 & -0.175 \\
\hline $\mathrm{Hb}$ & 0.014 & 0.063 & 0.009 & -0.036 \\
\hline \multicolumn{5}{|c|}{ Mean values of blood pressure and heart rate } \\
\hline Mean value of SBP & -0.077 & -0.116 & -0.219 & $0.349 *$ \\
\hline Mean value of DBP & -0.150 & -0.037 & -0.018 & 0.123 \\
\hline Mean value of $\mathrm{HR}$ & 0.026 & -0.192 & -0.172 & 0.227 \\
\hline \multicolumn{5}{|l|}{ Visit-to-visit variability of $S B P$} \\
\hline s.d. of SBP & 0.286 & $-0.327^{*}$ & $-0.496^{* * *}$ & $0.384^{*}$ \\
\hline Max-min SBP difference & $0.319^{*}$ & -0.297 & $-0.490 * * *$ & $0.410^{* *}$ \\
\hline
\end{tabular}

Abbreviations: $\mathrm{e}^{\prime}$, peak early diastolic mitral annular velocity; A, peak late diastolic transmitral flow; BMI, body mass index; DBP, diastolic brachial blood pressure; Hb, hemoglobin; $\mathrm{HDL}$,

high-density lipoprotein; $H R$, heart rate; $E$, peak early diastolic transmitral flow; E/A, the ratio of

$E$ to $A ; E / e^{\prime}$, the ratio of $E$ to $e^{\prime} ; L V$, left ventricular; LVMI, left ventricular mass index; SBP,

systolic brachial blood pressure; s.d., standard deviation.

${ }^{*} P<0.05,{ }^{* *} P<0.01,{ }^{* * *} P<0.001$.

E/e' was associated with both parameters of visit-to-visit SBP as well as mean SBP during the 1-year observation. Therefore, stepwise multiple regression analysis was performed to identify which parameters over the year were independently associated with $\mathrm{E} / \mathrm{e}^{\prime}$. The parameters that showed statistically significant correlations with diastolic function in univariate analyses (mean value of SBP, s.d. of SBP and maximumminimum SBP difference) are shown in Table 3. This analysis indicates only maximum-minimum SBP difference $(\beta$ coefficient $=0.410$, $P=0.009, r^{2}=0.168$ ) was independently associated with E/e' (Table 4).

\section{DISCUSSION}

The present study compared visit-to-visit variability and the mean values of SBP data over a 1-year observation period to identify the associations between echocardiographic parameters and CAVI in treated hypertensive patients. The data led us to the following conclusions: (1) the highest level of SBP was observed in November, and the lowest level of SBP was observed in August over a 1-year observation; (2) although visit-to-visit SBP variability over the 1-year period correlated with the mean SBP over the same year, the correlation was weak ( $r=0.353, P=0.025)$; (3) arterial stiffness as assessed by CAVI did not show statistically significant correlations with visit-tovisit variability parameters or mean SBP over the 1-year observation; (4) LV hypertrophy as assessed by LVMI did not show close correlations with visit-to-visit SBP variability parameters or the mean value of SBP; (5) in contrast, parameters of LV diastolic function were well correlated with the three parameters of SBP over the 1-year observation; and (6) among the parameters of SBP, maximum-minimum SBP difference (a variability parameter) demonstrated the closest correlation with parameters of LV diastolic function.

Seasonal variation in arterial blood pressures has previously been demonstrated; ${ }^{12-19}$ blood pressure is generally higher in the winter
Table 4 Multiple regression analysis of E/e' and significantly associated variables

\begin{tabular}{lccc}
\hline Independent variable & $\beta$-coefficient & t-value & P-value \\
\hline Max-min SBP difference & 0.410 & 2.774 & 0.009 \\
Mean value of SBP & 0.222 & 1.400 & 0.170 \\
s.d. of SBP & -0.107 & -0.205 & 0.839 \\
& Fratio=7.694 & $r^{2}=0.168$ & \\
& $(P=0.009)$ & \\
\hline
\end{tabular}

Abbreviations: $e^{\prime}$, peak early diastolic mitral annular velocity; E, peak early diastolic transmitral flow; E/e', the ratio of $E$ to $e^{\prime}$; Max-min, maximum-minimum; SBP, systolic blood pressure; s.d., standard deviation.

than in the summer. We previously reported the highest level of SBP is observed in February, and the lowest level of SBP is observed in August in healthy elderly subjects based on home measurements of blood pressures. ${ }^{31}$ In the present study, the highest level of SBP was observed in November, which is the beginning of winter. In other words, the month in which the highest level of SBP was observed was different from that in previous reports. ${ }^{12-19,31}$ However, the present study used the SBP at an outpatient clinic and included treated hypertensive patients. These differences in methods of blood pressure measurement and study participants may have caused this discrepancy between the present study and previous studies.

In the present study, s.d. of SBP correlated with the mean value of SBP $(r=0.353, P=0.025)$ (Table 2$)$. However, our data suggest the clinical significance of visit-to-visit SBP variability and that of mean SBP over a 1-year observation may be different. In the present study, we sought to elucidate the difference in vascular damage produced by the visit-to-visit SBP variability and the mean value of SBP by measuring CAVI, which can reflect the influence of hypertension on arterial stiffness. However, there were no statistically significant correlations between CAVI, the visit-to-visit SBP variability parameters and the mean SBP. Previous reports ${ }^{30,32}$ have established CAVI is not as influenced by blood pressure as brachial-ankle PWV is influenced. Therefore, brachial-ankle PWV might produce different results regarding correlations between arterial stiffness and visit-to-visit SBP variability and the mean SBP. Moreover, the population used in the present study was small. A previous study, ${ }^{33}$ which was performed by measuring PWV in a larger number of patients, reported a feasible link between arterial stiffness and seasonal blood pressure variation.

In the present study, we focused on differences in associations with cardiac damage between visit-to-visit SBP variability parameters and mean SBP. We found that neither visit-to-visit SBP variability nor mean SBP showed a close correlation with LVMI, which reflects LV hypertrophy in treated hypertensive patients. However, visit-to-visit SBP variability parameters and mean SBP showed close correlations with parameters of LV diastolic function. This finding may indicate that LV diastolic dysfunction rather than LV hypertrophy reflects cardiac damage in treated hypertensive patients. This may be because LV diastolic dysfunction occurs even in hypertensive patients without LV hypertrophy. Indeed, in previous studies mildly hypertensive patients demonstrated LV diastolic dysfunction without LV hypertrophy. ${ }^{34,35}$ In our study, the correlation was closer for the visit-to-visit SBP variability parameters than for mean SBP. These results indicate that visit-to-visit variability in SBP over a 1-year period may be a marker of LV diastolic dysfunction in treated hypertensive patients. Therefore, hypertensive patients with greater visit-to-visit variability of SBP may have a high risk of diastolic heart failure.

We could not determine the precise mechanism for the association between high visit-to-visit variability of SBP and LV diastolic 
dysfunction. The association between arterial stiffening and LV diastolic dysfunction (that is, arterial-ventricular coupling) has been proposed as one reason for LV diastolic heart failure with preserved LV systolic function. ${ }^{36,37}$ In the present study, hypertensive patients with LV diastolic dysfunction may have had increased arterial stiffness, which might have led to the increased SBP in response to seasonal variations, such as environmental temperature, over the 1-year observation period. Therefore, hypertensive patients with LV diastolic dysfunction may have shown greater visit-to-visit SBP variability over the 1-year observation. An association between arterial stiffening and LV diastolic dysfunction may have existed in the participants of the present study.

This study has several limitations. First, there was a significant correlation between the mean SBP and maximum-minimum SBP difference $(r=0.390, P<0.05)$ (Table 2$)$. Because both parametersmean SBP and maximum-minimum SBP difference-were used for the multiple regression analysis, there is the problem of multicollinearity. However, we selected study participants from two groups: (1) seven patients with lower mean SBP $(<129 \mathrm{~mm} \mathrm{Hg})$ and a greater maximum-minimum SBP difference $(\geqslant 30 \mathrm{~mm} \mathrm{Hg})$ and (2) seven patients with higher mean SBP $(\geqslant 129 \mathrm{~mm} \mathrm{Hg})$ and a smaller maximum-minimum SBP difference $(<30 \mathrm{~mm} \mathrm{Hg})$, and we compared the diastolic function between the two groups. Although E/A and E/ $\mathrm{e}^{\prime}$ did not differ between the two groups, the $\mathrm{e}^{\prime}\left(5.0 \pm 0.8 \mathrm{~cm} \mathrm{~s}^{-1}\right)$ in seven patients with lower mean SBP $(<129 \mathrm{~mm} \mathrm{Hg})$ and a greater maximum-minimum SBP difference $(\geqslant 30 \mathrm{~mm} \mathrm{Hg})$ was significantly lower (worse) than the $\mathrm{e}^{\prime}\left(6.7 \pm 1.2 \mathrm{~cm} \mathrm{~s}^{-1}\right)$ in seven patients with higher mean SBP $(\geqslant 129 \mathrm{~mm} \mathrm{Hg})$ and a smaller maximum-minimum SBP difference $(<30 \mathrm{~mm} \mathrm{Hg})$. This result suggests that high visit-tovisit variability is independently associated with $\mathrm{LV}$ diastolic dysfunction. Second, the seasonal variability of SBP was not very large in the present study because the s.d. of SBP was $11 \pm 5 \mathrm{~mm} \mathrm{Hg}$ (Table 1). Therefore, further studies are needed to establish clinical significance of association between seasonal SBP variability and LV diastolic dysfunction. Finally, we have no data regarding the effects of antihypertensive treatment on LV diastolic dysfunction and seasonal SBP variability because the present study was a cross-sectional design and did not determine the effects of antihypertensive treatments.

In conclusion, parameters of visit-to-visit variability of SBP showed a stronger correlation with LV diastolic dysfunction than did mean SBP over the 1-year observation. In particular, increased s.d. of SBP over the year was associated with LV diastolic dysfunction and may indicate a high risk for diastolic heart failure in treated hypertensive patients.

1 Diamond JA, Phillips RA. Hypertensive heart disease. Hypertens Res 2005; 28 : 191-202.

2 Kai H, Kuwahara F, Tokuda K, Imaizumi T. Diastolic dysfunction in hypertensive hearts: roles of perivascular inflammation and reactive myocardial fibrosis. Hypertens Res 2005; 28: 483-490.

3 Levy D, Garrison RJ, Savage DD, Kannel WB, Castelli WP. Prognostic implications of echocardiographically determined left ventricular mass in the Framingham Heart Study. N Engl J Med 1990; 322: 1561-1566.

4 Verdecchia P, Schillaci G, Borgioni C, Ciucci A, Gattobigio R, Zampi I, Reboldi G, Porcellati $C$. Prognostic significance of serial changes in left ventricular mass in essential hypertension. Circulation 1998; 97: 48-54.

5 Schillaci G, Pasqualini L, Verdecchia P, Vaudo G, Marchesi S, Porcellati C, de Simone G, Mannarino E. Prognostic significance of left ventricular diastolic dysfunction in essential hypertension. J Am Coll Cardiol 2002; 39: 2005-2011.

6 Redfield MM, Jacobsen SJ, Burnett Jr JC, Mahoney DW, Bailey KR, Rodeheffer RJ. Burden of systolic and diastolic ventricular dysfunction in the community: appreciating the scope of the heart failure epidemic. JAMA 2003; 289: 194-202.

7 Senni M, Tribouilloy CM, Rodeheffer RJ, Jacobsen SJ, Evans JM, Bailey KR, Redfield MM. Congestive heart failure in the community: a study of all incident cases in Olmsted County, Minnesota, in 1991. Circulation 1998; 98: 2282-2289.
8 Jessup M, Brozena S. Heart failure. N Engl J Med 2003; 348: 2007-2018.

9 Owan TE, Redfield MM. Epidemiology of diastolic heart failure. Prog Cardiovasc Dis 2005; 47: 320-332.

10 Lakatta EG. Arterial pressure and aging. Int J Cardiol 1989; 25 (Suppl 1): S81-S89.

11 Lakatta EG. Similar myocardial effects of aging and hypertension. Eur Heart J 1990; 11(Suppl G): 29-38.

12 Hata T, Ogihara T, Maruyama A, Mikami H, Nakamaru M, Naka T, Kumahara Y, Nugent CA. The seasonal variation of blood pressure in patients with essential hypertension. Clin Exp Hypertens 1982; 4: 341-354.

13 Woodhouse PR, Khaw KT, Plummer M. Seasonal variation of blood pressure and its relationship to ambient temperature in an elderly population. J Hypertens 1993; 11: 1267-1274.

14 Kristal-Boneh E, Harari G, Green MS. Seasonal change in 24-hour blood pressure and heart rate is greater among smokers than nonsmokers. Hypertension 1997; 30(3 Part 1): 436-441.

15 Chifamba J, Mufunda J, Sigola LB, Matenga JA, Sparks HV. Effect of variation in environmental temperature on blood pressure: is it important? Cent Afr J Med 1998; 44: 37-40.

16 Nakajima J, Kawamura M, Fujiwara T, Hiramori K. Body height is a determinant of seasonal blood pressure variation in patients with essential hypertension. Hypertens Res 2000; 23: 587-592.

17 Sothern RB. Circadian and circannual characteristics of blood pressure self-measured for 25 years by a clinically-healthy man. Chronobiologia 1994; 21: 7-20.

18 Paschalis-Purtak K, Pucilowska B, Kabat M, Sznajderman M. Clinical evaluation of $24 \mathrm{~h}$ ambulatory monitoring of blood pressure under various environmental conditions (home and work versus hospital). Blood Press Monit 1998; 3: 289-294.

19 Imai Y, Munakata M, Tsuji I, Ohkubo T, Satoh H, Yoshino H, Watanabe N, Nishiyama A, Onodera N, Kato J, Sekino M, Aihara A, Kasai Y, Abe K. Seasonal variation in blood pressure in normotensive women studied by home measurements. Clin Sci (Lond) 1996; 90: 55-60.

20 Khaw KT, Barrett-Connor E, Suarez L, Criqui MH. Predictors of stroke-associated mortality in the elderly. Stroke 1984; 15: 244-248.

21 Barrett-Connor E, Suarez L, Khaw K, Criqui MH, Wingard DL. Ischemic heart disease risk factors after age 50. J Chronic Dis 1984; 37: 903-908.

22 Rothwell PM, Howard SC, Dolan E, O'Brien E, Dobson JE, Dahlöf B, Sever PS, Poulter NR. Prognostic significance of visit-to-visit variability, maximum systolic blood pressure, and episodic hypertension. Lancet 2010; 375: 895-905.

23 Rothwell PM. Limitations of the usual blood-pressure hypothesis and importance of variability, instability, and episodic hypertension. Lancet 2010; 375: 938-948.

24 Wallerson DC, Devereux RB. Reproducibility of echocardiographic left ventricular measurements. Hypertension 1987; 9(2 Part 2): ॥6-II18.

25 Teichholz LE, Kreulen T, Herman MV, Gorlin R. Problems in echocardiographic volume determinations: echocardiographic-angiographic correlations in the presence of absence of asynergy. Am J Cardiol 1976; 37: 7-11.

26 Nishimura RA, Appleton CP. 'Diastology': beyond E and A. J Am Coll Cardiol 1996; 27: 372-374.

27 Nishimura RA, Tajik AJ. Evaluation of diastolic filling of left ventricle in health and disease: Doppler echocardiography is the clinician's Rosetta Stone. J Am Coll Cardiol 1997; 30: 8-18.

28 Sohn DW, Chai IH, Lee DJ, Kim HC, Kim HS, Oh BH, Lee MM, Park YB, Choi YS, Seo JD, Lee YW. Assessment of mitral annulus velocity by Doppler tissue imaging in the evaluation of left ventricular diastolic function. J Am Coll Cardiol 1997; 30: 474-480.

29 Ommen SR, Nishimura RA, Appleton CP, Miller FA, Oh JK, Redfield MM, Tajik, AJ. Clinical utility of Doppler echocardiography and tissue Doppler imaging in the estimation of left ventricular filling pressures: a comparative simultaneous Doppler-catheterization study. Circulation 2000; 102: 1788-1794.

30 Shirai K, Utino J, Otsuka K, Takata M. A novel blood pressure-independent arterial wall stiffness parameter; cardio-ankle vascular index (CAVI). J Atheroscler Thromb 2006; 13: 101-107.

31 Kimura T, Senda S, Masugata H, Yamagami A, Okuyama H, Kohno T, Hirao T, Fukunaga M, Okada H, Goda F. Seasonal blood pressure variation and its relationship to environmental temperature in healthy elderly Japanese studied by home measurements. Clin Exp Hypertens 2010; 32: 8-12.

32 Satoh N, Shimatsu A, Kato Y, Araki R, Koyama K, Okajima T, Tanabe M, Ooishi M, Kotani K, Ogawa Y. Evaluation of the cardio-ankle vascular index, a new indicator of arterial stiffness independent of blood pressure, in obesity and metabolic syndrome. Hypertens Res 2008; 31: 1921-1930.

33 Youn JC, Rim SJ, Park S, Ko YG, Kang SM, Choi D, Ha JW, Jang Y, Chung N. Arterial stiffness is related to augmented seasonal variation of blood pressure in hypertensive patients. Blood Press 2007; 16: 375-380.

34 Aeschbacher BC, Hutter D, Fuhrer J, Weidmann P, Delacretaz E, Allemann Y. Diastolic dysfunction precedes myocardial hypertrophy in the development of hypertension. Am J Hypertens 2001; 14: 106-113.

35 Chang NC, Lai ZY, Wang TC. Enalapril does not improve left ventricular diastolic dysfunction in young and mild hypertensives without concomitant hypertrophy. Am J Hypertens 1996; 14: 909-914.

36 Desai AS, Mitchell GF, Fang JC, Creager MA. Central aortic stiffness is increased in patients with heart failure and preserved ejection fraction. J Card Fail 2009; 15: 658-664.

37 Borlaug BA, Olson TP, Lam CS, Flood KS, Lerman A, Johnson BD, Redfield MM. Global cardiovascular reserve dysfunction in heart failure with preserved ejection fraction. J Am Coll Cardiol 2010; 56: 845-854. 